\title{
Nucleation kinetics in a supercooled metallic glass former
}

\author{
F. Puosi* and A. Pasturel \\ Univ. Grenoble Alpes, CNRS, Grenoble INP ${ }^{\mathrm{b}}$, SIMaP, F-38000 Grenoble, France
}

\begin{abstract}
We use molecular dynamics simulations to shed light on the mechanism underlying crystal nucleation in a supercooled metallic glass former characterized by a concurring crystal and amorphous local order based on icosahedral symmetry. At a crossover temperature, well below the melting point, we find that the supercooled phase exhibits glassy dynamics which includes a breakdown of the Stokes-Einstein relation and the emergence of spatially heterogeneous dynamics. In addition, we show that the origin of these phenomena can be related to a structural heterogeneity caused by the increase of icosahedral symmetry upon cooling. We also reveal that crystal nucleation occurs close to the glass transition and takes place in regions of high icosahedral symmetry, which can be interpreted by a strong reduction of the crystalliquid interfacial energy. This scenario provides a framework for testing the estimation of the nucleation time according to the classical nucleation theory. More specifically, our findings allow to quantify how the heterogeneous character of the supercooled phase affects the predictions of this theory.
\end{abstract}

Keywords: metallic glass, supercooled liquid region, nucleation, heterogeneity, molecular dynamics

\footnotetext{
${ }^{\mathrm{b}}$ Institute of Engineering Univ. Grenoble Alpes

* francesco.puosi@df.unipi.it; Present address: Dip. di Fisica "Enrico Fermi”, Università di Pisa, Largo

B.Pontecorvo 3, I-56127 Pisa, Italy
} 


\section{INTRODUCTION}

The ultimate fate of a liquid upon cooling below its equilibrium melting temperature, $T_{F}$, whether it crystallizes or becomes a glassy state - depends on how easily the crystal can nucleate. In this supercooling regime, the characteristic of time scale for crystal nucleation, the nucleation time $\tau_{n}$, is mainly described by the so-called time-temperature-transformation diagram (TTT diagram) which exhibits a "nose" shape [1]. In other words, the nucleation time displays a sharp minimum, $\tau_{n}\left(T_{\text {nose }}\right)$ at the "nose" temperature $T_{\text {nose }}$, located below $T_{F}$. It is widely believed that crystallization mechanism changes from a nucleation process above $T_{\text {nose }}$ to a growth-controlled process below [2]. The TTT diagram is also often used to discuss the glass forming ability (GFA) of a supercooled liquid. To form a glass, the liquid must be cooled from $T_{F}$ to below $T_{\text {nose }}$ in a time less than $\tau_{n}\left(T_{\text {nose }}\right)$ [3]. This defines a critical cooling rate $R_{c} \sim\left(T_{F}-T_{\text {nose }}\right) / \tau_{n}\left(T_{\text {nose }}\right)$, above which a liquid avoids crystallization and form a metastable supercooled liquid that freezes into a non-equilibrium glassy state at $T_{g}$ when structural relaxation is too long to be observable within experimental time scales.

Yet, crystallization rather than the glass transition may still occur in this deep supercooling region. Indeed, the structural relaxation time, $\tau_{\alpha}$ and the nucleation time $\tau_{n}$, which are well separated close to $T_{F}$ (being $\tau_{\alpha}$ much shorter), become comparable with decreasing temperatures below $T_{\text {nose }}$. Such a behavior defines a "lower metastability limit" (LML) or a "homogeneous nucleation limit" (HNL), below which the supercooled liquid ceases to be observable because it nucleates before it can equilibrate [4-6].

A reasonable description for the temperature-dependence of the structural relaxation time below $T_{F}$ [7] is given by the Vogel-Fulcher-Tamman law (VFT), $\tau_{\alpha}(T)=\tau_{0} \exp \left[B T_{0} /\left(T-T_{0}\right)\right]$ where $\tau_{0}$ and $B$ are constants and $T_{0}$ the so-called VFT temperature. $B$ is known as the fragility index, which is negatively correlated with the fragility [8]. Namely a smaller $B$ in the VTF law corresponds to a more fragile liquid and it is associated to a steep increase of $\tau_{\alpha}(T)$ with decreasing temperature.

An estimate of the nucleation time can be obtained from the classical nucleation theory (CNT) [9] which considers that the initial homogeneous disordered liquid and the final ordered crystal phases are the only key ingredients of nucleation. For homogeneous nucleation, the nucleation

time is given by $\tau_{n}(T)=K \exp \left(\Delta G^{*} / R T\right)$, where $R$ is the gas constant and $\Delta G^{*}$ the free energy cost of a crystal nucleus at the top of the nucleation barrier. Here $K$ is a kinetic prefactor propor- 
tional to the characteristic time of material transport controlling crystallization, namely the inverse of the liquid diffusion, $D^{-1}$. Tanaka [5] argued that HNL depends on how one treats the kinetic prefactor. Assuming that $D^{-1}$ is proportional to $\tau_{\alpha}$ via the Stokes-Einstein (SE) relation (here we use the structural relaxation time as a proxy for viscosity), CNT suggests that $\tau_{n}(T)$ is always greater than $\tau_{\alpha}$, which implies that the equilibration of the supercooled liquid is always obtained prior to nucleation or in other words the HNL does not exist. In this context, Tanaka pointed out that a liquid will exhibit a HNL if $D^{-1}$ is strongly decoupled to $\tau_{\alpha}$ in the supercooled regime and the SE relation breaks down. Cavagna et al. [6] obtained similar conclusions by incorporating the SE breakdown into CNT through viscoelastic effects. In contrast, Zanotto et al observed the occurence of a HNL without the breakdown of SE relation [10], suggesting that the latter is not a necessary condition.

Some studies [7] attributed the breakdown of the SE relation to the emergence of dynamic heterogeneities (DHs) which provide an explicit connection between spatial fluctuations and relaxation kinetics [11]. Specifically, the breakdown has been associated to the particles in fast regions while particles in slow regimes obey the SE relation [12]. However, some other studies proposed that it is not necessary to invoke DHs to explain the breakdown of SE relation [13-15] and also evidenced that particles in both fast and slow regions can deviate from the SE relation [13, 16]. Therefore, despite intensive efforts, the physical factors that control these dynamic phenomena that characterize the deep supercooled region remain elusive. More particularly, the structural signature of the dynamical arrest at the origin of the glass transition is still under debate [17].

For metallic liquids, it was conjectured that local configurations with a high degree of five-fold symmetry (FFS) or local icosahedral symmetry may explain the stability of supercooled states against crystallization [18]. Recently, the degree of local FFS was shown to be a structural indicator to interpret the temperature-dependence of dynamic properties during supercooling in metallic melts [19]. The increase in FFS-based motifs in the supercooling regime has been also used to present the glass transition as a frustration against crystallization. A popular scenario is based on geometrical frustration associated with the fact that FFS-based motifs would form on cooling a continuous ordered phase if the space is curved [20]. In the Euclidian space, that growth is frustrated, giving rise to the formation of limited domains only. An alternative scenario is proposed [21], based on frustration effects of non-extendable FFS- based motifs on crystal-like ordering, which prevent crystallization and favor glass formation. In both approaches, FFS-based motifs act as inhibitors of crystallization. 
Yet, an open question is to investigate the physical factors that control the interplay between crystallization and glass formation when FFS-based motifs can be also regarded as structural motifs linked to a crystalline ground state. To address this question, we focus on the $\mathrm{Cu}-\mathrm{Zr}$ system since it is known to exhibit a good glass-forming ability over a wide composition range [22] but also to crystallize in an ordered phase at the specific composition $\mathrm{Cu}_{5} \mathrm{Zr}$, characterized by a remarkable fraction of local icosahedral symmetry. In this work, we use large-scale molecular dynamics simulations for the $\mathrm{Cu}_{5} \mathrm{Zr}$ metallic liquid to investigate the role of icosahedral symmetry in the interplay between crystallization and glass formation in its deep supercooling regime.

We find that crystal nucleation can be identified from the determination of the TTT diagram with a "nose" temperature located in the deep supercooling region, just above the glass transition temperature. Dynamics slowdown in this region is associated with the presence of DHs and the breakdown of the SE relation which occur at a crossover temperature located well above the "nose" temperature. By exploring the evolution of local ordering during cooling we show that this crossover temperature corresponds to the increase of local icosahedral symmetry in slow regions of DHs. We also evidence that local icosahedral symmetry develops during crystallization. All these results allow to propose a scenario in which slow regions of DHs act as crystal nucleation precursors. Finally these findings are used to test the validity and the predictive power of CNT.

\section{COMPUTATIONAL DETAILS}

We consider a model for the $\mathrm{Cu}_{5} \mathrm{Zr}$ alloy with atoms interacting via en embedded-atom model (EAM) potential [23]. Molecular Dynamics (MD) simulations are carryed out using LAMMPS molecular dynamics software [24]. The system consists of a total number of 23328 atoms, 19440 $\mathrm{Cu}$ and $3888 \mathrm{Zr}$ atoms, contained in a box with periodic boundary conditions in three dimensions. The initial configurations are equilibrated at $2000 \mathrm{~K}$ for $5 \mathrm{~ns}$ followed by rapid quenching $\left(10^{11} \mathrm{~K} / \mathrm{s}\right)$ to $500 \mathrm{~K}$ in NPT ensemble. During quenching, the cell size is adjusted to give zero pressure and configurations at different temperatures are collected. More specifically, dynamic and structural properties are computed from $1500 \mathrm{~K}$ to $825 \mathrm{~K}$, in a range where liquid equilibrium is established on time scale much shorter than the nucleation time. This prevents any interference of nucleation events on the accurate determination of liquid properties.

For dynamic properties, we focus on self-diffusion, structural relaxation and the emergence of DHs. Self-diffusion coefficients are evaluated from the mean-square displacement of each 
component $i(i=\mathrm{Cu}$ or $\mathrm{Zr})$ :

$$
R_{i}^{2}(t)=\frac{1}{N_{i}} \sum_{k=1}^{N_{i}}\left\langle\left[\mathbf{r}_{k}\left(t+t_{0}\right)-\mathbf{r}_{k}\left(t_{0}\right)\right]^{2}\right\rangle
$$

where $\mathbf{r}_{k}$ represents the position of atom $k$ of specie $i$ at time $t$. The angular brackets represent the average over time origins $t_{0}$. The self-diffusion coefficient $D_{i}$ is then determined from the long time limit of $R_{i}^{2}(t)$, namely $D_{i}=\lim _{t \rightarrow \infty} R_{i}^{2}(t) / 6 t$

Structural relaxation times are obtained by the self-intermediate scattering function which is defined for each component by:

$$
F_{s, i}(q, t)=\frac{1}{N_{i}}\left\langle\sum_{k=1}^{N_{i}} \exp \left[i \mathbf{q} \cdot\left(\mathbf{r}_{k}\left(t+t_{0}\right)-\mathbf{r}_{k}\left(t_{0}\right)\right)\right]\right\rangle
$$

The structural relaxation time of component $i, \tau_{\alpha}^{i}$, is defined as the time needed for the corresponding self-intermediate scattering function $F_{s, i}(q, t)$ to be equal to $1 / e$ at a wave vector $q=q_{\max }$, corresponding to the main peak in the static structure factor [25].

To quantify the emergence of DHs, we consider the non-Gaussian parameter (NGP) [26] defined by:

$$
\alpha_{2}(t)=3 R^{4}(t) / 5\left[R^{2}(t)\right]^{2}-1
$$

where $R^{2}(t)$ and $R^{4}(t)=N_{i}^{-1} \sum_{k=1}^{N_{i}}\left\langle\left[\mathbf{r}_{k}\left(t+t_{0}\right)-\mathbf{r}_{k}\left(t_{0}\right)\right]^{4}\right\rangle$ are respectively the mean-square and mean-quadruple displacements of one component. $\alpha_{2}(t)$ exhibits a peak at a characteristic time scale $t^{*}$, signaling that the degree of DHs is maximum at $t^{*}$. We indicate as $\alpha_{2}^{\max }$ the maximum value of $\alpha_{2}(t)$.

To investigate DHs more in details, we resort to the method introduced by Widmer-Cooper and Harrowell [27] based on the so-called isoconfigurational (IC) ensemble. Here 100 NVT trajectories are simulated starting from the same initial configuration but with different initial velocities, drawn randomly from a Maxwell-Boltzmann distribution. Typically, each simulation lasts for a time at least of the order of the structural relaxation time to allow DHs to evolve. Atomic mobility is evaluated via the IC averaged mean square displacement:

$$
\left\langle\Delta r_{j, \alpha}^{2}(t)\right\rangle_{i c}=\left\langle\left|\mathbf{r}_{j, \alpha}(\Delta t)-\mathbf{r}_{j, \alpha}(0)\right|^{2}\right\rangle
$$

where $\mathbf{r}_{j, \alpha}(0)$ and $\mathbf{r}_{j, \alpha}(\Delta t)$ are the positions of the $j$ th atoms at the beginning and after a time $\Delta t$ in the $\alpha$ th trajectory and the average $\langle\ldots\rangle_{i c}$ is taken over all the IC trajectories. 
For structural properties, we compute the orientational order parameter, following the prescription proposed by Steinhardt et al. [28]. First, we define the set of neighbors of the atom $j$ as all the atoms $k$ within a cutoff distance $r_{c}$ given by the first minimum in the radial distribution function. The corresponding vectors joining neighbors, $\mathbf{r}_{j k}$, are called bonds. The orientation of the vector $\mathbf{r}_{j k}$ determines the polar and azimuthal angle $\theta\left(\mathbf{r}_{j k}\right)$ and $\phi\left(\mathbf{r}_{j k}\right)$. The structure around the particle $j$ is characterized by the $2 l+1$ quantities:

$$
q_{l, m}(j)=\frac{1}{N_{b}(j)} \sum_{k=1}^{N_{b}(j)} Y_{l, m}\left(\theta\left(\mathbf{r}_{j k}\right), \phi\left(\mathbf{r}_{j k}\right)\right)
$$

where $Y_{l, m}$ are spherical harmonics and $N_{b}(j)$ is the number of bonds of particle $j$. The bond orientational order (BOO) parameters are defined by the rotationally invariant combinations:

$$
q_{l}(j)=\left[\frac{4 \pi}{2 l+1} \sum_{m=-l}^{l}\left|q_{l, m}(j)\right|^{2}\right]^{1 / 2}
$$

Following previous works $[29,30]$, we focus on $q_{l}$ with $l=6$, which is known to be very sensitive to any kind of crystallization.

\section{RESULTS}

\section{A. Time-temperature-transformation (TTT) diagram}

To quantify the crystal nucleation kinetics, we monitor the evolution of the potential energy of distinct configurations during the isothermal annealing at different temperatures, ranging from $1000 \mathrm{~K}$ to $800 \mathrm{~K}$. This range of temperatures is well below the experimental melting temperature, i.e. $T_{F}=1300 \mathrm{~K}$, and then corresponds to supercooled conditions. Fig. 1(a) shows the time dependence of the potential energy of one representative sample between $860 \mathrm{~K}$ and $800 \mathrm{~K}$. For each temperature, the crystal nucleation is detected by a significant drop in the potential energy with annealing time. Interestingly, the onset time of potential energy drops exhibits the typical shape of the TTT diagram with minimum nucleation time around $837 \mathrm{~K}$. In the inset of Fig. 1(a), we show the mean nucleation time given by the average over at least six independent realizations. Note that simulation cells are large enough to observe more than one nucleation event. Uncer-

tainties are computed as the standard deviation of the mean. We can estimate $T_{\text {nose }} \approx 830 \mathrm{~K}$. Then, above $T_{\text {nose }}$, we note that the potential energy displays a nearly constant variation before 

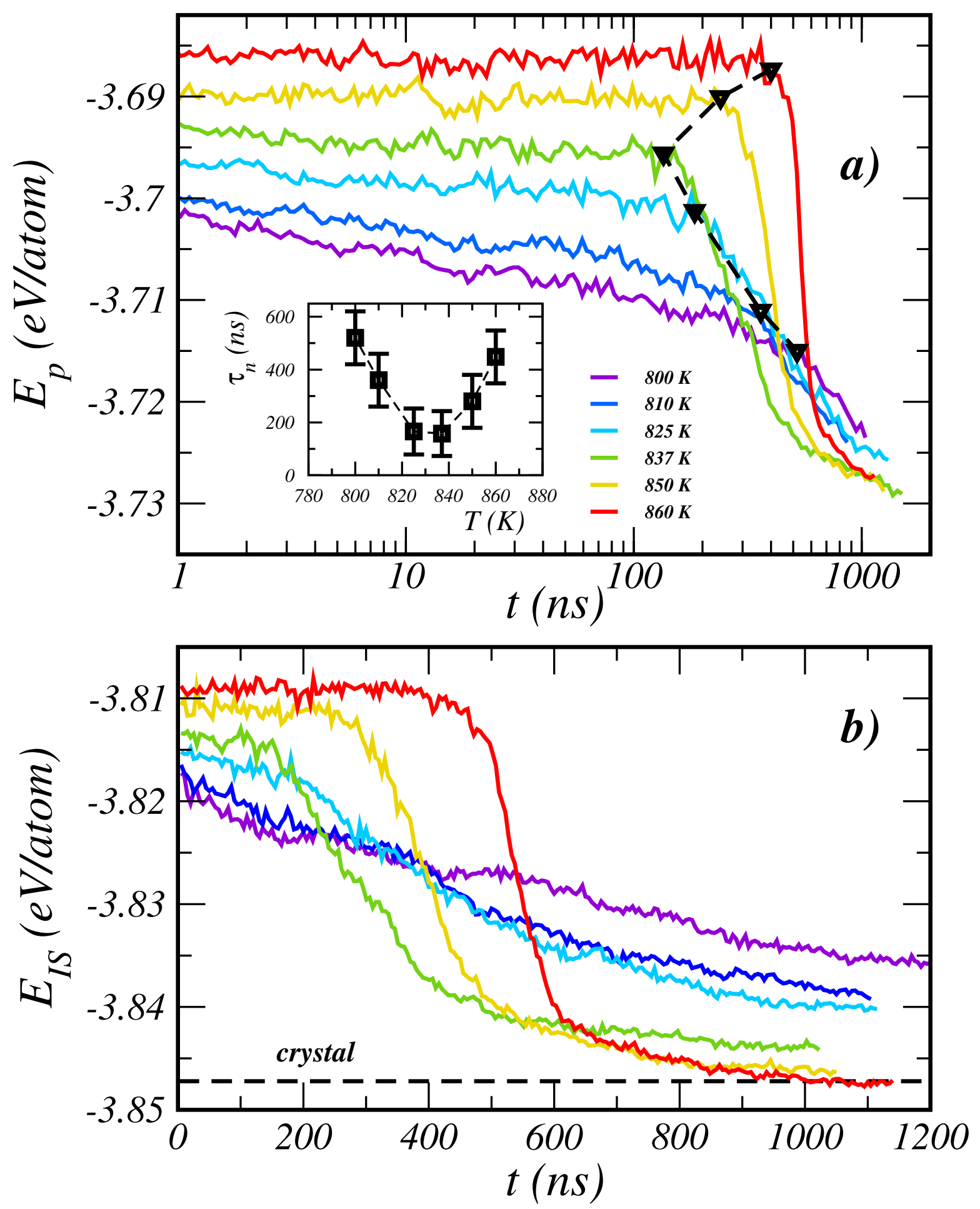

FIG. 1. Panel a): time evolution of the potential energy of a sample along isotherms at selected temperatures. Open triangles mark the nucleation times, defined as the onset time of potential energy drops. Inset: temperature dependence of the mean nucleation time $\tau_{n}$ as obtained by the average over at least six independent sample. Panel b): corresponding time dependence of the inherent structure energy. Dashed line indicates the energy of the $\mathrm{Cu}_{5} \mathrm{Zr}$ crystal. 
dropping abruptly at a time which defines the nucleation time as the latest time at which the liquid contains no crystalline nuclei. Below $T_{\text {nose }}$, the potential energy starts to decrease slowly during isothermal annealing, which indicates that nucleation will be concomitant to transient aging induced by the cooling process at low temperatures. Yet, we can still detect the nucleation time from a well-pronounced drop of the potential energy. In addition, we show in Fig. 1(b) the evolution of the inherent structure energy $E_{I S}$, i.e., the mean energy of the local minima in the potential energy explored by the system [31]. For $T>T_{n o s e}, E_{I S}$ of the supercooled liquid drops to a value similar to that of the $\mathrm{Cu}_{5} \mathrm{Zr}$ crystal, which is a first indication of the $\mathrm{Cu}_{5} \mathrm{Zr}$ crystallization. For $T<T_{\text {nose }}$ the difference between $E_{I S}$ of the supercooled liquid and that of $\mathrm{Cu}_{5} \mathrm{Zr}$ remains significant even at the end of our annealing process, which may indicate a sluggish crystal growth [32]. Indeed, the comparison with the energy of the glassy state, $E_{I S} \approx 3.82 \mathrm{eV} /$ atom, estimated after cooling the liquid to $500 \mathrm{~K}$, confirms that a fraction of non-crystallized phase still exists and becomes more and more important with decreasing temperature. Another indication of such dynamic slowing down is the proximity of the glass transition, since its estimation from the inherent structure energy during the cooling process yields $T_{g} \sim 750 \mathrm{~K}$.

\section{B. Dynamic properties in the supercooled region}

To analyze more in details dynamic properties of the $\mathrm{Cu}_{5} \mathrm{Zr}$ liquid in its supercooled regime, we first evaluate the temperature dependence of the structural relaxation time of each component, $\tau_{\alpha}^{i}$. Here, the supercooled state means a stationary metastable state whose evolution starts from an initial random configuration and is monitored for a time interval during which no crystal nucleation occurs. At high temperatures, $\tau_{\alpha}^{i}$ changes almost linearly with $1 / T$ as shown in Fig. 2(a), indicating an Arrhenius behavior. At lower temperatures, $\tau_{\alpha}^{i}$ increases drastically and deviates from the linear behavior below a crossover temperature, $T_{S} \sim 1100 \mathrm{~K}$ (for each component), located well below $T_{F}$. Fig. 2(a) indicates that the VFT law gives a good representation of the calculated structural relaxation times in the investigated temperature range. More specifically, we mention that fitted values, $B=1.33(1)$ and $T_{0}=700(2) \mathrm{K}$, do not depend on the component (the reported uncertainties account for the small differences of the fitting parameters for $\mathrm{Cu}$ and $\mathrm{Zr}$ data). The small value of $B$ points to the fragile character of the $\mathrm{Cu}_{5} \mathrm{Zr}$ alloy. A rapid comparison of the temperature dependence of $\tau_{\alpha}^{i}$ with that of $\tau_{n}$ (see Fig. 1(a)) shows that above $T_{n o s e}, \tau_{n}$ remains greater than $\tau_{\alpha}^{i}$ by at least three orders of magnitude, indicating that the liquid equilibrium is well 
established prior to crystal nucleation. This comparison within the framework of CNT will be discussed more in details below.
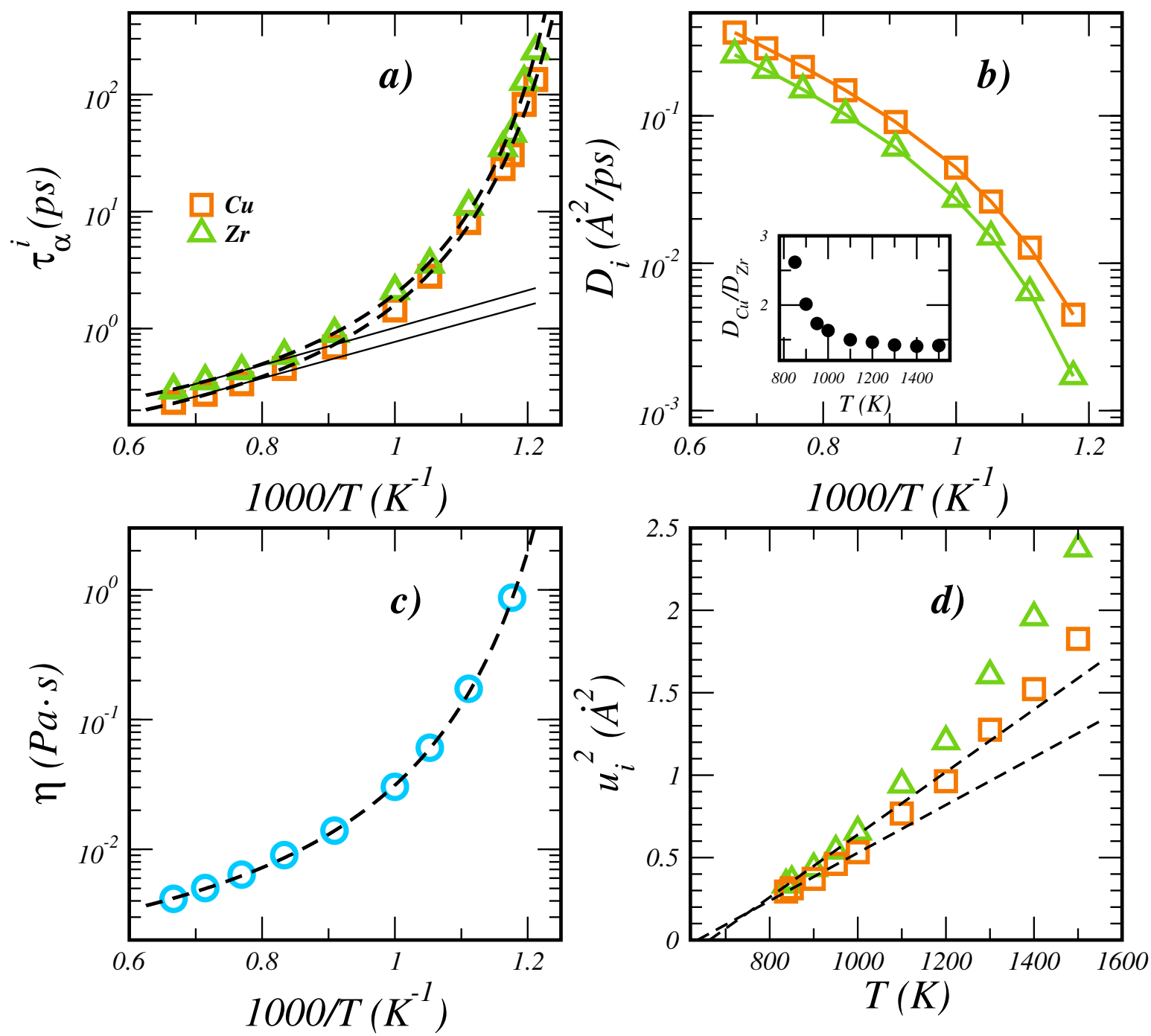

FIG. 2. Panels a) and b): Arrhenius plot of the structural relaxation time $\tau_{\alpha}^{i}$ (panel (a)) and self-diffusion coefficient $D_{i}$ (panel (b)) of $\mathrm{Cu}$ and $\mathrm{Zr}$ atoms. Inset of panel b): temperature dependence of the ratio between $\mathrm{Cu}$ and $\mathrm{Zr}$ diffusivity. Panel c): Arrhenius plot of the viscosity. Full lines in a) are fitted with the Arrhenius law $\tau_{\alpha}^{i}=A_{i} \exp \left(E_{i} / T\right)$. Dashed lines in b) and c) are fitted with the Vogel-Fulcher-Tammann (VFT) laws $X=A \exp \left(B T_{0} /\left(T-T_{0}\right)\right)$ where $X=\tau_{\alpha}^{i}, \eta$. Panel d): temperature dependence of the Debye-Waller factor $\left\langle u^{2}\right\rangle$ of $\mathrm{Cu}$ and $\mathrm{Zr}$ atoms. The dashed lines correspond to the linear fits in the low temperature range.

Next we report the temperature dependence of calculated self-diffusion coefficients, $D_{i}$, for each component. From Fig. 2(b), we see that their inverse temperature dependence displays an 
Arrhenius-type behavior only at high temperatures while we detect a sharp deviation from the Arrhenius fit below the crossover temperature $T_{S} \sim 1100 \mathrm{~K}$. Interestingly, the $D_{\mathrm{Cu}} / D_{\mathrm{Zr}}$ ratio shown in the inset of Fig. 2(b) points out the decoupling of component diffusion below $T_{S}$.

Then, we consider viscous and vibrational dynamics. In Fig. 2(c) the temperature dependence of the viscosity $\eta$ is shown. In agreement with previous observations for relaxation, VFT law provides a good description of viscosity data below $T_{S}$. Note that the fitted parameters $B$ and $T_{0}$ are similar to those extracted from the fit of structural relaxation times. Vibrational dynamics is described by the Debye-Waller (DW) factor $u^{2}$ corresponding to the mean square vibrational amplitude of atoms. Here, we define $u_{i}^{2}=R_{i}^{2}(t=1 \mathrm{ps})$; this definition ensures that, at sufficiently low temperature, $u_{i}^{2}$ corresponds to the plateau value of the mean square displacement, denoting particle caging [33]. Figure 2(d) displays the evolution of $u_{i}^{2}$ as a function of temperature for both components. We observe that the extrapolated $u_{i}^{2}$ seem to vanish at the VFT temperature $T_{0} \approx 700 \mathrm{~K}$. In addition significant deviations of the DW factor from the low temperature linear behavior become apparent below $T_{S}$.

Using the determination of self-diffusion coefficients and structural relaxation times, we can test the validity of the $\mathrm{SE}$ relation. It is well admitted that $D_{i} / T$ and $\tau_{\alpha}^{i}$ follow the $\mathrm{SE}$ relation, $D_{i} / T \propto 1 / \tau_{\alpha}^{i}$ at high temperatures. Fig. 3(a)-(b) shows that $D_{i} / T$ as a function of $\tau_{\alpha}^{i}$ obeys the SE relation above $T_{S}$ for both components. Below $T_{S}$, we observe a deviation from the $\mathrm{SE}$ relation and $D_{i} / T$ and $\tau_{\alpha}^{i}$ follow a fractional SE relation, i.e. $D_{i} / T \propto\left(\tau_{\alpha}^{i}\right)^{-\kappa}$ with $\kappa=0.65$ and 0.70 respectively for $\mathrm{Cu}$ and $\mathrm{Zr}$. Such a result confirms that relaxation and dynamics are significantly changed as $T_{S}$ is traversed.

To go further in the analysis of dynamic properties in the supercooled region, we examine the emergence of DHs. The latter can be revealed from the non-Gaussian parameter by following the evolution of its maximum value $\alpha_{2, \max }$ as a function of temperature. Namely, dramatic increase of $\alpha_{2, \max }$ above a value of 0.2 indicates the rapid growing of DHs. The change of $\alpha_{2, \max }$ with temperature can be illustrated more clearly by its first derivative against temperature, $d \alpha_{2, \max } / d T$. In Fig. 4(a)-(b) we display $\alpha_{2, \max }$ and $d \alpha_{2, \max } / d T$ for each component. For both $\mathrm{Cu}$ and $\mathrm{Zr}$, our findings evidence an onset of DHs at the crossover temperature $T_{S}$, below which they grow with an increasing rate.

Thus, we can consider that $T_{S}$ corresponds to a strong evolution in dynamic properties of the $\mathrm{Cu}_{5} \mathrm{Zr}$ liquid through the decoupling of diffusion, the Arrhenius-to-non-Arrhenius transition of structural relaxation time, the presence of fractional SE relation and the emergence of DHs. To 

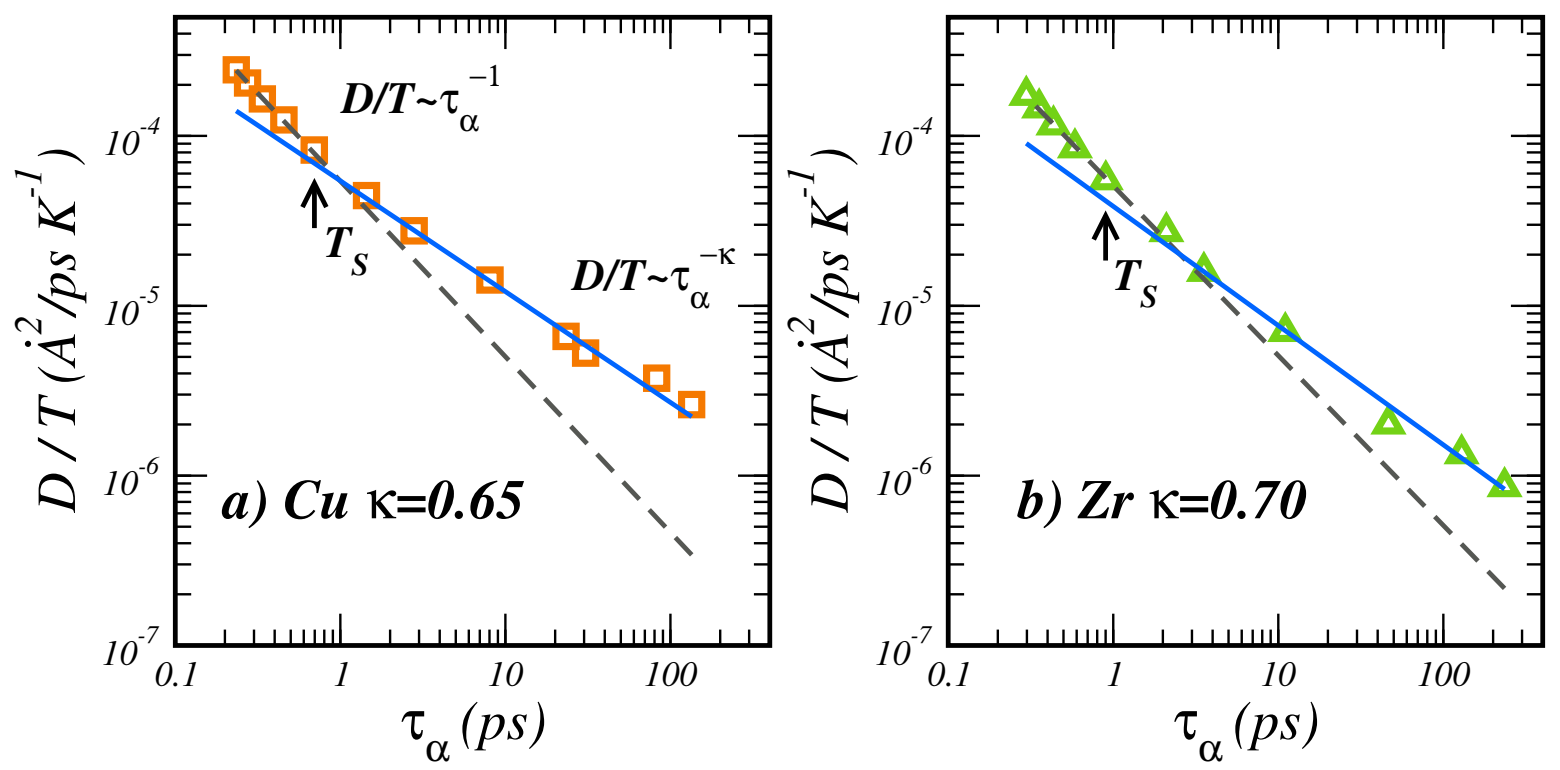

FIG. 3. Ratio $D / T$ as a function of $\tau_{\alpha}$ for $\mathrm{Cu}$ (panel (a)) and $\mathrm{Zr}$ (panel (b)) atoms. The dashed and full lines correspond to the SE relation $D_{i} / T \sim\left(\tau_{\alpha}^{i}\right)^{-1}$ and fractional SE relation $D_{i} / T \propto\left(\tau_{\alpha}^{i}\right)^{-\kappa}$ respectively.

reveal the underlying structural signature of dynamic crossover and to explore its relationship with crystal nucleation, we now investigate the structure of the $\mathrm{Cu}_{5} \mathrm{Zr}$ liquid during quenching and isothermal annealing.
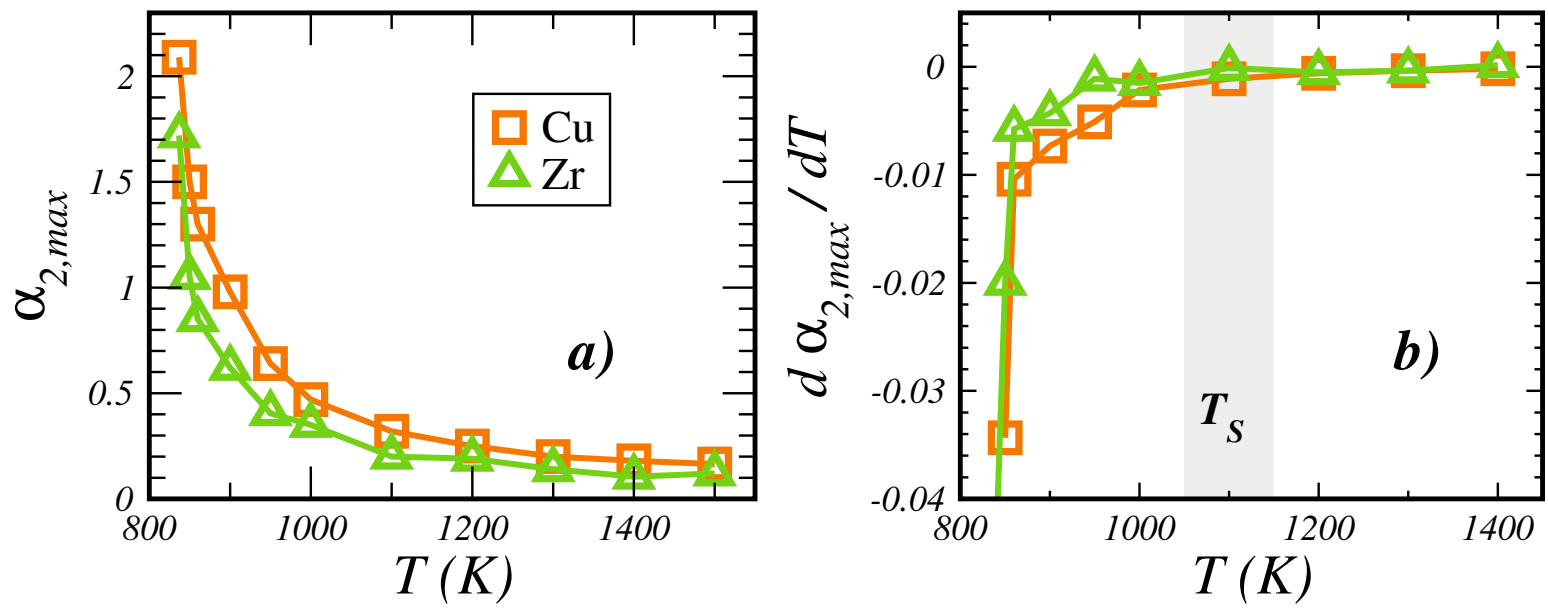

FIG. 4. Panel a): temperature dependence of the maximum value of the NGP, $\alpha_{2, \max }$, for $\mathrm{Cu}$ and $\mathrm{Zr}$ atoms. Panel b): corresponding temperature derivative $d \alpha_{2, \max } / d T$ as a function of temperature. The shaded region marks the onset temperature $T_{S}$, denoting the appearance of DHs. 


\section{Structural signatures of dynamic crossover phenomena and crystal nucleation}

Here atomic structures in the $\mathrm{Cu}_{5} \mathrm{Zr}$ liquid are characterized by the BOO parameter $q_{6}$ which can be also used to identify solid-like particles in liquids [34]. Fig. 5(a)-(b) shows the temperature dependence of the $\mathrm{BOO}$ parameter for each component. For the most abundant $\mathrm{Cu}$ component (see Fig. 5(a)) the BOO parameter at high temperatures is broadly distributed and quite similar to that of an equilibrated liquid in the Lennard-Jones system [34]. However, at low temperatures,


FIG. 5. Panel a): distribution of the local orientational order parameter $q_{6}$ of $\mathrm{Cu}$ atoms for selected temperature values. Vertical dashed line corresponds to the $q_{6}$ value of $\mathrm{Cu}$ atoms with icosahedral symmetry in $\mathrm{Cu}_{5} \mathrm{Zr}$ crystal. Inset: temperature dependence of the fraction of $\mathrm{Cu}$ atoms with icosahedral local orientational order, i.e. with a $q_{6}$ in the shaded region of the main panel. Vertical line marks the onset temperature $T_{S}$. Panel b): Corresponding $q_{6}$ distribution of $\mathrm{Zr}$ atoms. Vertical dashed line marks the $q_{6}$ value of $\mathrm{Zr}$ atoms in $\mathrm{Cu}_{5} \mathrm{Zr}$ crystal.

a second peak appears at a $q_{6}$ value $\approx 0.62$. This tendency becomes more pronounced at deeper supercooling and quite remarkably, the steep increase of this $\mathrm{Cu}$ fraction occurs at the crossover temperature $T_{S}$ as shown in the inset of Fig. 5(a). Another interesting feature is that this $q_{6}$ value is close to that obtained in a perfectly icosahedral configuration, namely $q_{6}=0.66$. In addition, we emphasize that $80 \%$ of $\mathrm{Cu}$ atoms in the $\mathrm{Cu}_{5} \mathrm{Zr}$ crystal have an icosahedral environment and thus are characterized by $q_{6}=0.66$ [35]. In Fig. 5(b) the BOO parameter of Zr component is 
narrowly distributed around a peak value $q_{6} \sim 0.17$ and does not present any significant change as a function of temperature. We note also that this $q_{6}$ value is close to that of $\mathrm{Zr}$ atoms in the $\mathrm{Cu}_{5} \mathrm{Zr}$ crystal, namely $q_{6} \sim 0.14$.

From these findings, we can conclude that the steep increase of the $\mathrm{BOO}$ parameter of $\mathrm{Cu}$ atoms around $q_{6} \sim 0.62$ at $T_{S}$ can be considered as a signature of the underlying link between dynamic crossover and local structural ordering. To deeply explore this coupling, we present in Fig. 6 the BOO distribution at a temperature close to $T_{n o s e}$, for only $\mathrm{Cu}$ atoms with the lowest and highest mobility in DHs i.e. the $5 \%$ of atoms with the lowest and highest values of the dynamic propensity as defined by Eq. (5). The mobile fraction has a broad $q_{6}$ distribution, similar to the global distribution shown in Fig. 5(a) except the icosahedral peak at $q_{6} \sim 0.62$. On the opposite, the distribution of the sluggish fraction is different from the global one and presents a bimodal character: the icosahedral peak is enhanced with respect to the global one at the expenses of the broad peak whose width decreases notably. Then, from Fig. 6, we clearly establish that $\mathrm{Cu}$ atoms with icosahedral symmetry are located in the domains of low propensity, namely the slow fraction of DHs.

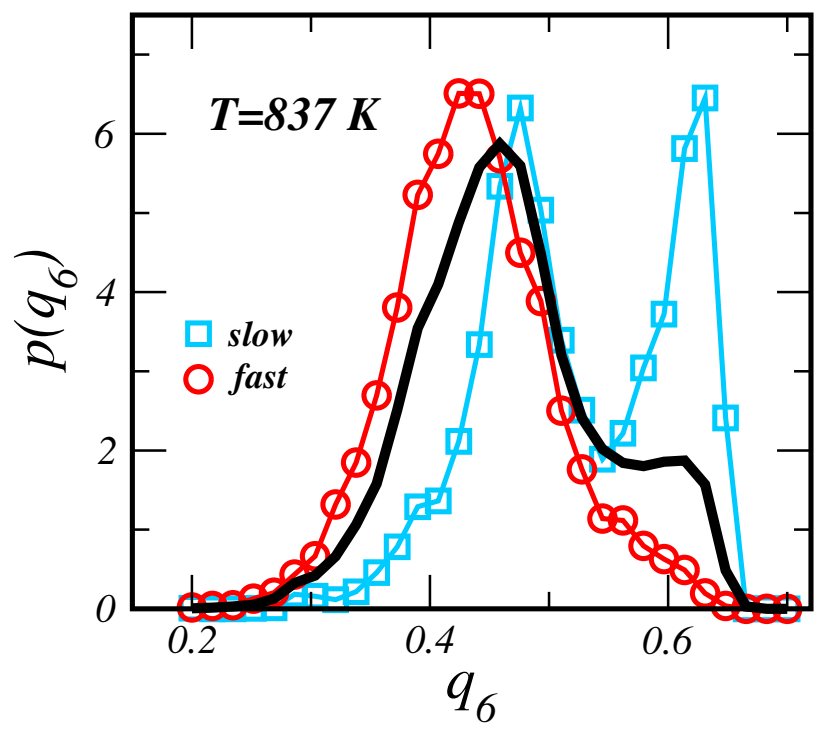

FIG. 6. For isothermal annealing at $T=837 \mathrm{~K}$, distribution of $q_{6}$ restricted to slow and fast $\mathrm{Cu}$ atoms in DHs, compared to the distribution for all $\mathrm{Cu}$ atoms (bold line).

To investigate the role of the $\mathrm{BOO}$ parameter in nucleation process, we examine its temporal evolution during isothermal annealing. Figure 7(a) shows the mean BOO parameter $\overline{q_{6}}$ of $\mathrm{Cu}$ atoms for the six temperatures investigated. We find that for all the temperatures $\overline{q_{6}}$ exhibits a 

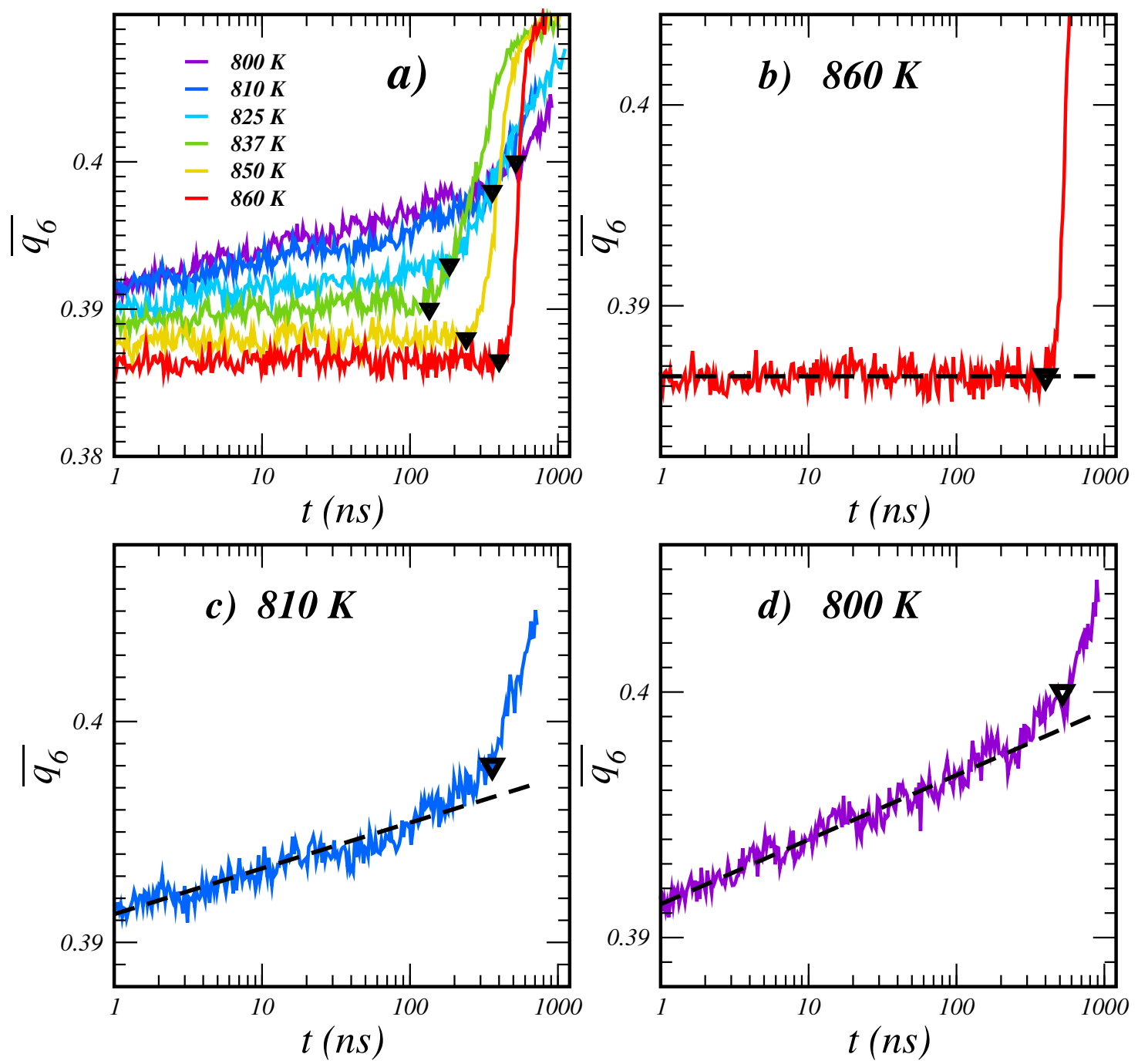

FIG. 7. Panel a): time evolution of the mean $\mathrm{BOO}$ parameter $\overline{q_{6}}$ of $\mathrm{Cu}$ atoms along isothermes at selected temperatures. Open triangles mark the nucleation times, defined as the onset time of potential energy drops and shown in Fig. 1. Panels c)-d): for representative temperature values, we show that the nucleation time identifies a change in the time evolution of $q_{6}$.

change in its temporal evolution, which is more pronounced at high temperatures (above $T_{\text {nose }}$ ) than at low ones (below $T_{\text {nose }}$ ), as seen in Fig. 7(b)-(d). We also emphasize that the onset of this change is well signaled by the nucleation time as defined from the energy drops shown in Figure 1. This confirms that the weak changes seen for the low-T annealing isothermes can be ascribed to nucleation occurring in the system.

Previous observations indicate that the nucleation process can be related to the time evolution of the BOO parameter. To go further, we inspect the time dependence of the $q_{6}$ distribution along 
the isotherm at a temperature close to $T_{\text {nose }}$. In Fig. 8(a) we observe an increase of the icosahedral peak at a time which corresponds to the onset of nucleation, namely $t=160 \mathrm{~ns}$. Note that this increase is even more rapid at later times, as evidenced in Fig. 8(b) which shows that the associated fraction of $\mathrm{Cu}$ atoms double during crystallization process. Such a behavior can be related to the growth of the $\mathrm{Cu}_{5} \mathrm{Zr}$ crystal which gives a positive feedback for the increase of icosahedral symmetry through its $80 \%$ of $\mathrm{Cu}$ atoms with icosahedral environment. Further two additional peaks develop at small $q_{6}$ values. The peak at $q_{6} \sim 0.2$ can be referred to the other $20 \% \mathrm{Cu}$ atoms of $\mathrm{Cu}_{5} \mathrm{Zr}$ crystal, which are characterized by $q_{6} \sim 0.192$. The other peak at $q_{6} \sim 0.3$ is less obvious to interpret. It could be related to the presence of defects in the crystallized structure, as already observed for other metallic systems [36].

To identify more carefully solid clusters associated with the crystal nucleation, namely prenuclei and nuclei, we use the BOO parameter $q_{6}$ as initially proposed by Frenkel et al. [34]. Note that in our system, the complex structure of the $\mathrm{Cu}_{5} \mathrm{Zr}$ crystal makes the identification of crystal nuclei a critical task, to a greater extent than in systems nucleating into simple structures. In particular, we must discriminate between atoms with icosahedral environment belonging or not to crystal nuclei. For this reason, we adopt the following criteria. We identify a $\mathrm{Cu}$ atom as "solid-like" if i) it is characterized by a $q_{6}$ parameter close to 0.62 , ii) it has more than four of its $\mathrm{Cu}$ neighbors with the same condition on $q_{6}$, and iii) its displacement on a time scale typical of diffusion motion is smaller than a threshold, fixed to the half of the mean inter-particle distance. This latter condition corresponds to an atom unable to diffuse, performing a rattling motion around its mean position. The solid cluster is defined by adding to each of these "solid-like" $\mathrm{Cu}$ atoms their first neighbors. Then we apply a cluster analysis to measure its size. In Fig. 8(c)-(d), we present the time dependence of such solid clusters, $N_{s} c$, and the size of the largest one, $N_{1}$, during the isothermal annealing at $T \sim T_{\text {nose }}$. First, at the early stages of the annealing, we observe fluctuations in the nucleation process with the presence of few solid clusters which repeatedly rise, grow and dissolve as they do not reach the critical nucleus size. At $t \approx 160 \mathrm{~ns}$, we find that the largest cluster overcomes the critical size, estimated at $1-2 \mathrm{~nm}$, to grow rapidly at later times. We note that this determination of the nucleation time is coherent with that given by the drop of the potential energy (see Fig. 1).

Figures 9 and 10 illustrate the nucleation process and the growth of nuclei at two different temperatures, $T=860 \mathrm{~K}$ (Fig.9) and $T=800 \mathrm{~K}$ (Fig.10), above $T_{\text {nose }}$ and below $T_{\text {nose }}$ respectively. We note that the nucleation and the growth processes are considerably different. More specifically, 

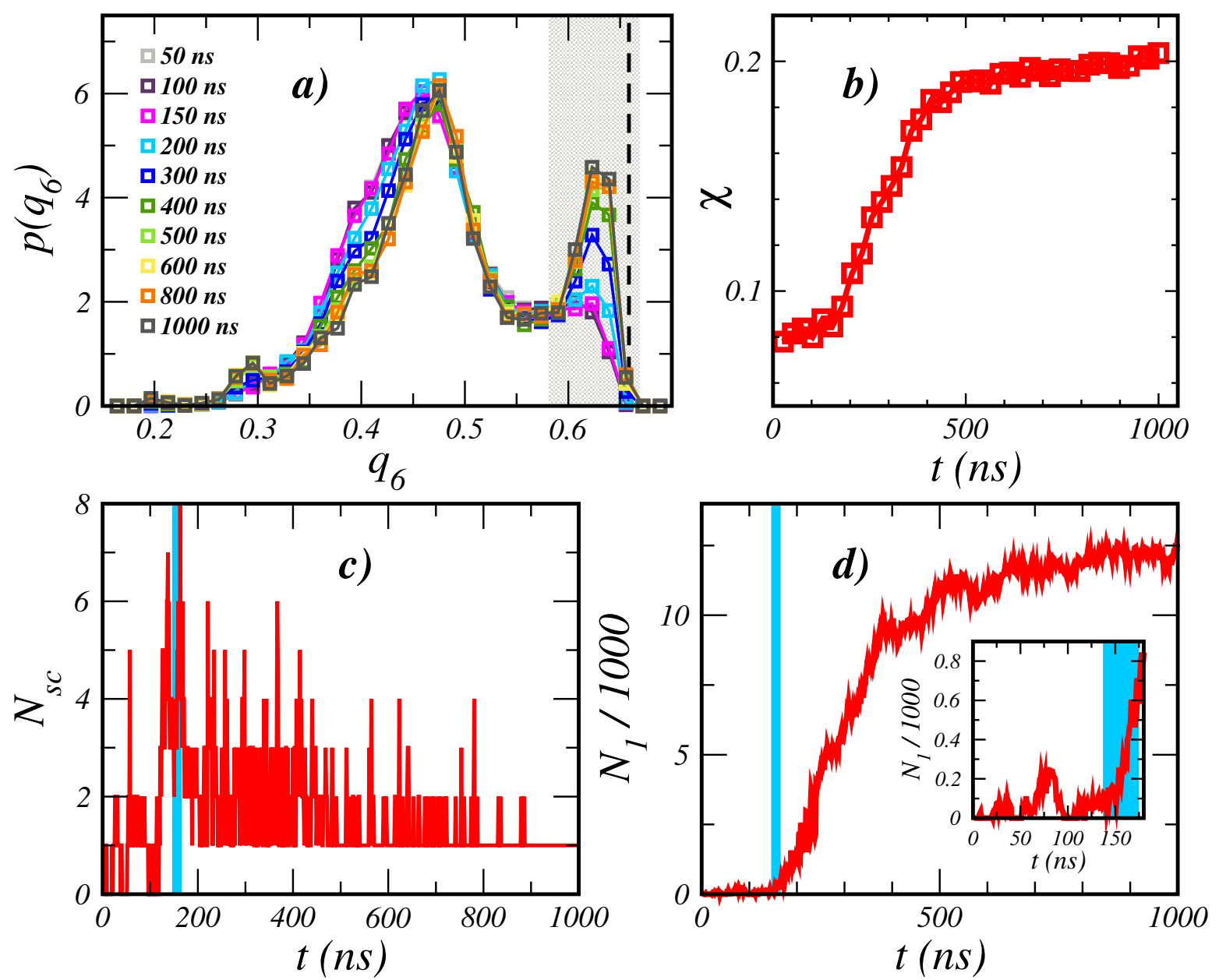

FIG. 8. Evolution of structural indicators along the isotherm at $T=837 \mathrm{~K}$. Panel a): evolution of the $q_{6}$ distribution for $\mathrm{Cu}$ atoms. Panel $\mathrm{b}$ ): corresponding time dependence of the fraction of $\mathrm{Cu}$ atoms with icosahedral local orientational order. Panel c): time dependence of the number of solid clusters $N_{s c}$. Panel d): population of the largest cluster $N_{1}$. The inset is a magnification at the onset of crystal nucleation. Vertical lines in c) and d) mark the nucleation time $\tau_{n}$.

at $T=860 \mathrm{~K}$ at the onset of nucleation a single nucleus is detected, which at later times grows rapidly. In contrast, for $T=800 \mathrm{~K}$ we report a larger number of nuclei and a slower growth rate. We argue that the number of nuclei and their surface fraction affect also the growth process: the more the crystal nuclei are formed, the slower the systems crystallize. Indeed, such a behavior is in line with our findings in Fig. 7(b)-(d): at low temperatures $\overline{q_{6}}$ displays a change in its time evolution less pronounced than at high temperatures. It can be also related to experimental findings [2] which evidence a change in the crystallization mechanism from a nucleation process above $T_{\text {nose }}$ to a growth-controlled process below. However, we emphasize that the understanding of the 
$390 \mathrm{~ns}$

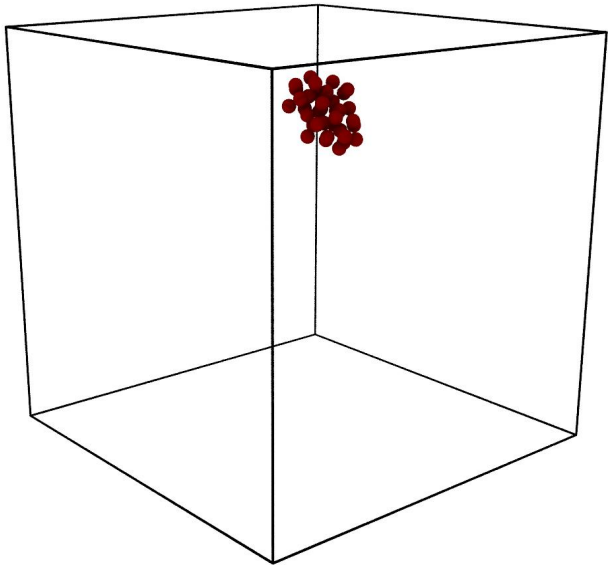

500 ns

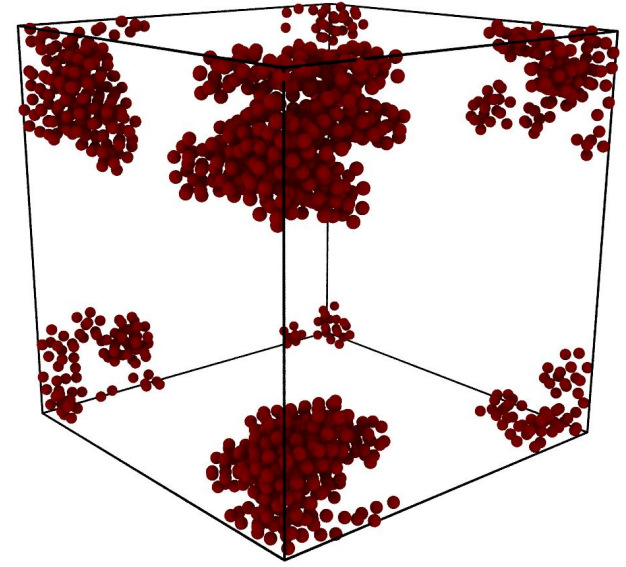

450 ns

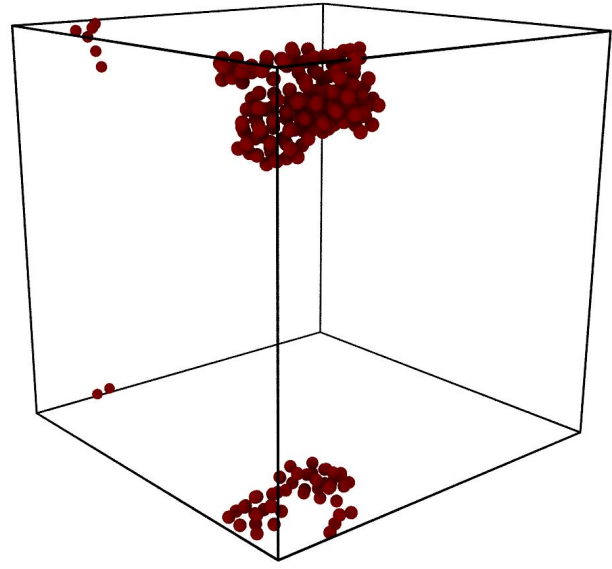

600 ns

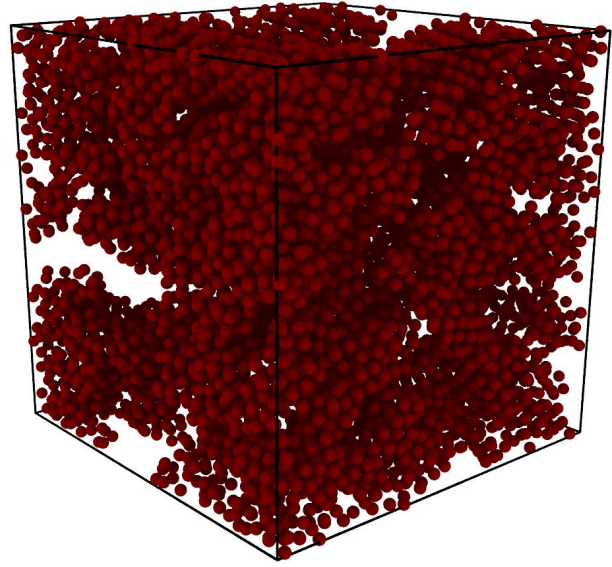

FIG. 9. Crystal nuclei for selected configurations along an isotherm at $T=860 \mathrm{~K}$, above $T_{\text {nose }}$. Atoms are colored according to a cluster analysis: in the specific case only one cluster is detected.

crystallization process in this deep supercooled system requires a more detailed analysis which is beyond the scope of this work.

\section{DISCUSSION}

We have demonstrated that the supercooled $\mathrm{Cu}_{5} \mathrm{Zr}$ liquid displays local icosahedral ordering which develops in slow regions of DHs upon cooling. Local icosahedral order was also found to induce the nucleation process of the crystalline phase during isothermal annealing in the deeply supercooled region where DHs exist. An intuitive conclusion is that the nucleation process starts in slow regions of DHs. To firmly establish this connection, we compare at $T \sim T_{\text {nose }}$ the spatial distribution of slow regions and crystal-like ones at different times, spanning from the pre-nucleation 
420 ns

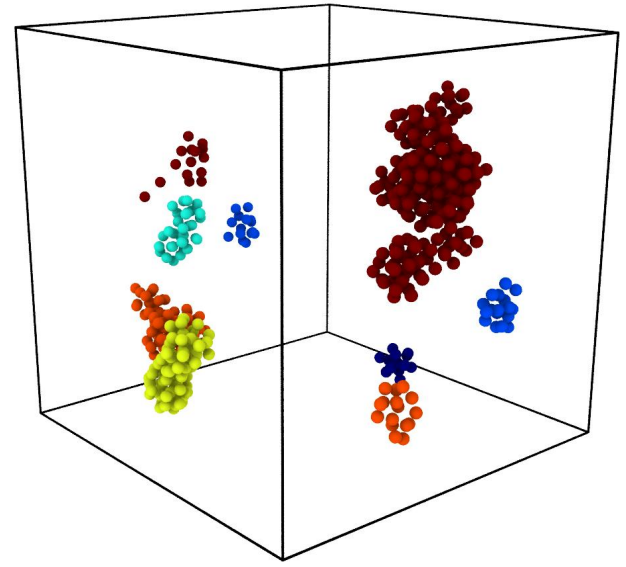

$780 \mathrm{~ns}$

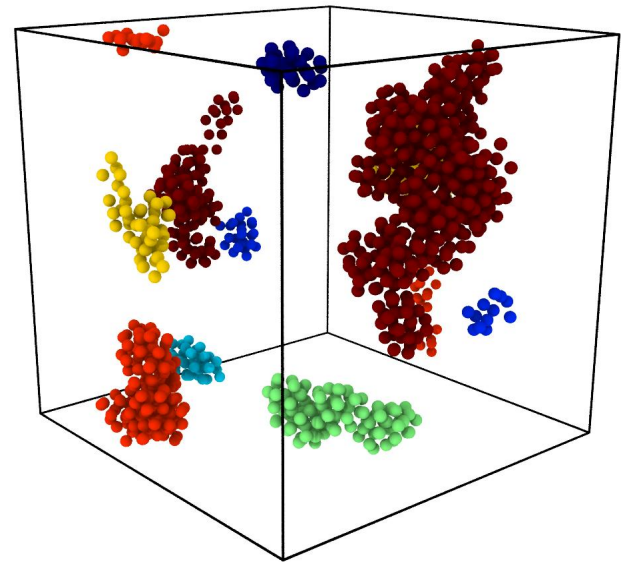

550 ns

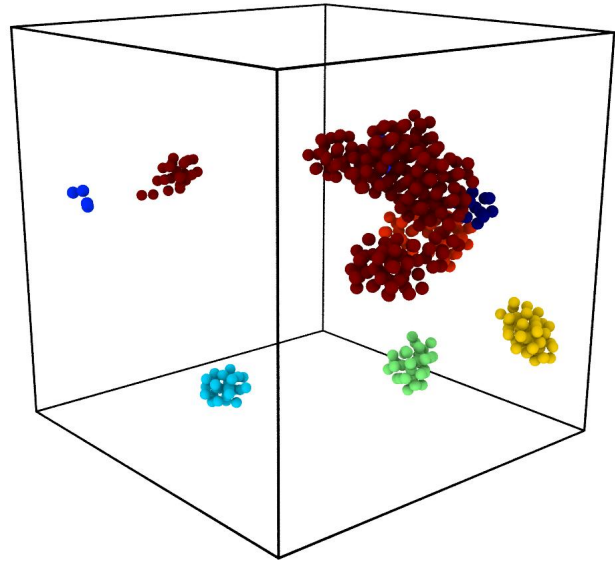

1000 ns

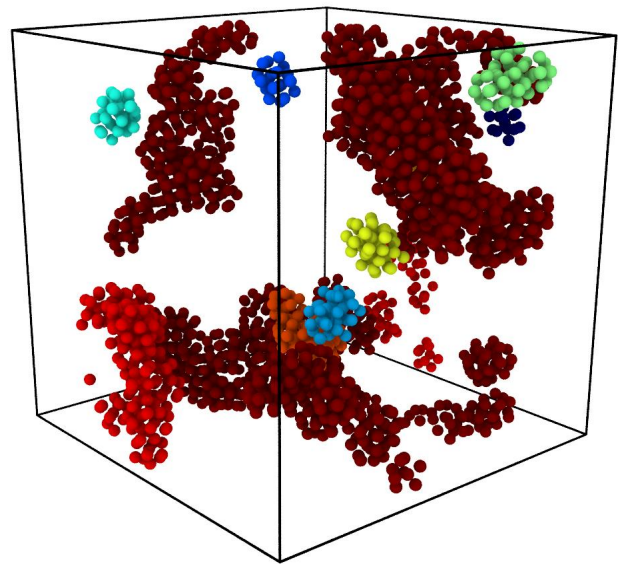

FIG. 10. Crystal nuclei for selected configurations along an isotherm at $T=800 \mathrm{~K}$, below $T_{\text {nose }}$. Atoms are colored according to a cluster analysis: distinct colors for atoms belonging to different clusters.

regime (50ns and 80ns) where no or few sub-critical nuclei exist to the first stages of crystallization (160ns and 200ns). First, Fig. 11 displays the slow regions defined by the $5 \%$ slowest atoms in DHs; we can see that the slowest atoms are not randomly distributed and tend to aggregate to form large regions. Next, during the isothermal annealing, we observe a clear correlation between slow regions of DHs and regions where the crystal nucleation is starting, namely the occurrence of sub-critical and stable crystal nuclei. Thus this correlation suggests that the physical mechanism behind the nucleation process of $\mathrm{Cu}_{5} \mathrm{Zr}$ in the supercooled regime is a matching of the bond orientational symmetry between slow regions of DHs and the crystal phase. The relevance of this scenario of symmetry-matching induced preferential nucleation in slow regions of DHs is supported by the fact that more than 100 nucleation events observed in this study all occur selectively 
in slow regions. A similar link between crystal nucleation kinetics and DHs in a supercooled liquid was reported by Gupta et al. [37], who showed that below a given crossover temperature the sizes of critical nuclei and the nucleation rate are controlled by the size of cooperatively rearranging regions (CRRs), i.e., the size scale of relaxation in a dynamically heterogeneous liquid.


FIG. 11. Correlation between crystal nuclei (colored particles) and the slow regions of dynamical heterogeneities (transparent particles) for selected configurations along an isotherm at $T=837 \mathrm{~K}$, close to $T_{\text {nose }}$.

Within CNT, this influence of preexisting order on the nucleation of the crystal phase in liquid can be understood in terms of the interfacial energy, $\gamma$, between the liquid and solid phases: the reduction of the interfacial energy significantly reduces the free-energy barrier for crystal nucleation. Assuming that the entropic contribution to $\gamma$ is dominant $[38,39]$, we can express the interfacial 
energy as $\gamma=\alpha \Delta S_{F} /\left(N_{A} V_{M}^{2}\right)^{1 / 3}$ where $\alpha$ is a temperature-independent quantity that provides a measure of the configurational entropy of the interface and $\Delta S_{F}$ the entropy of fusion. $V_{M}$ denotes the molar volume and $N_{A}$ Avogadro number. Experimental and theoretical estimates of $\alpha$ show a systematic dependence on the topological structure of the solid phase [40]. As a matter of fact, $\alpha$ decreases as the degree of tetrahedral and icosahedral order in the solid phase increases. For instance, for the polytetrahedral phases $\alpha \sim 0.4$, significantly smaller than those found for simple crystal with cubic or hexagonal structures, namely $\alpha^{f c c / h p c} \sim 0.85$ and $\alpha^{b c c} \sim 0.70$ [40]. Note that such a behavior is also consistent with experimental studies in supercooled metallic systems $[41,42]$, which is another strong evidence of the importance of tetrahedral and icosahedral order in these systems.

To have a more quantitative understanding, we can evaluate the free-energy barrier $\Delta G^{*}$ for the formation of a nucleus of critical size. According to nucleation theory, $\Delta G^{*}=16 \pi \gamma^{3} / 3 \Delta G_{V}^{2}$ with $\Delta G_{V}=G_{L}-G_{S}$, the difference of Gibbs free energy per unit volume of liquid $\left(G_{L}\right)$ and solid $\left(G_{S}\right)$ phases. The previous expression is derived assuming that critical nuclei have spherical shapes. This first approximation seems reasonable for the present case, if looking to Fig. 11 where crystal nuclei appear as rather compact objects. For metallic systems, the driving force for nucleation $\Delta G_{V}$ can be approximated by $\Delta G_{V}=\Delta S_{F} \Delta T V_{M}^{-1}$ with $\Delta T=T_{F}-T$ [43]. With the previous approximation for the interfacial energy, we find that $\Delta G^{*}$ obeys a temperature dependence based on CNT of the type $\Delta G^{*}=\mathcal{C} T^{3} / \Delta T^{2}$ with $\mathcal{C}=\frac{16 \pi}{3 N_{A}} \alpha^{3} \Delta S_{F}$. The entropy of fusion $\Delta S_{F}$ is determined to be $12.82 \mathrm{~J} \mathrm{~mol}^{-1} \mathrm{~K}^{-1}$ at $T_{F}=1290 \mathrm{~K}$ [44].

In $\mathrm{CNT}$, the so-called nose temperature is a result of the competition between thermodynamic and kinetic effects, respectively the increase of the driving force for crystallization and the decrease of mobility upon cooling. Thus we can express the temperature dependence of the nucleation time as

$$
\tau_{n}^{C N T}(T)=K^{\prime} D^{-1}(T) \exp \left(\frac{\mathcal{C}^{\prime} T^{2}}{\Delta T^{2}}\right)
$$

taking into account approximations used to determine $\Delta G^{*}$ and $\mathcal{C}^{\prime}$ given by $\mathcal{C} / k_{B}$ with $k_{B}$ the Boltzmann's constant. $K^{\prime}$ depends only weakly on temperature if compared to the exponential term and $D^{-1}(T)$. The temperature dependence of $D^{-1}(T)$ is extracted from MD simulations via the fractional SE relation, which links $D(T)$ with $\tau_{\alpha}(T)$ (see Fig. 3), and assuming a VFT-type dependence on the temperature for $\tau_{\alpha}$ (see Fig. 2). Accordingly, Eq. 7 becomes:

$$
\tau_{n}^{C N T}(T)=\frac{K^{\prime \prime}}{T} \exp \left(\kappa \frac{B T_{0}}{T-T_{0}}+\frac{\mathcal{C}^{\prime} T^{2}}{\Delta T^{2}}\right)
$$


First, to compare the temperature dependence of $\tau_{n}^{C N T}(T)$ with that given by MD simulations, we choose $K^{\prime \prime}$ to obtain $\tau_{n}^{C N T}=\tau_{n}$ at $T=837 \mathrm{~K}$. Further, taking into account the high degree of local icosahedral ordering in the $\mathrm{Cu}_{5} \mathrm{Zr}$ crystal, we choose the $\alpha$ value to be 0.4 in agreement with previous estimations [40]. Note that a change of \pm 0.1 does not modify our conclusions. The result of this comparison is shown in Fig. 12 (dash-dotted line) and evidences that the functional form of CNT satisfactory describes the MD- based nucleation times at different temperatures. In Fig. 12, we also compare the temperature dependence of $\tau_{n}^{C N T}(T)$ to that of $\tau_{\alpha}(T)$. Below $T=837 \mathrm{~K}$, we see that the gap between $\tau_{n}^{C N T}$ and $\tau_{\alpha}(T)$ is closing rapidly, suggesting that the supercooled liquid reaches a HNL in the vicinity of $770 \mathrm{~K}$, which is only $20 \mathrm{~K}$ higher than $T_{g}$. We stress that the temperature extrapolation of $\tau_{n}^{C N T}$, which is necessary to obtain this conclusion, is only about $40 \mathrm{~K}$.

Interestingly, we can quantify the role played by the SE breakdown in the occurrence of HNL as discussed by Tanaka [5]. To do so, we also estimate the temperature dependence of the nucleation time using the data for $D(T)$ related to $\tau_{\alpha}(T)$ via the SE relation (dashed line in Fig. 3). Within this assumption, Eq. 8 is modified as:

$$
\tau_{n}^{C N T / S E}(T)=\frac{K^{\prime \prime}}{T} \exp \left(\frac{B T_{0}}{T-T_{0}}+\frac{\mathcal{C}^{\prime} T^{2}}{\Delta T^{2}}\right)
$$

We see in Fig. 12 that below $T=800 \mathrm{~K}, \tau_{n}^{C N T / S E}(T)$ is as much as an order of magnitude larger than $\tau_{n}^{C N T}$ and that $\tau_{n}^{C N T / S E}$ and $\tau_{\alpha}$ are more widely separated and converging less rapidly than $\tau_{n}^{C N T}$ and $\tau_{\alpha}$. Then, this result suggests that if the SE relation were obeyed, HNL would be shifted to lower $T$, in agreement with Tanaka's conclusions obtained for a strong metallic glass former.

Next, we seek to determine the nucleation time in CNT from the steady-state nucleation rate described in the conventional form [45-47] by:

$$
I_{s s}=N_{0} \sqrt{\frac{\sigma}{k_{B} T}} \frac{D(T)}{a} \exp \left(-\frac{\Delta G^{*}}{k_{B} T}\right)
$$

where $N_{0}$ is the number of potential nucleation sites and $a$ the typical interatomic spacing obtained from the number density. The nucleation time is then given by $\tau_{n}^{C N T}=\left(I_{s s} V_{s y s}\right)^{-1}$ with $V_{\text {sys }}$ the system volume [48]. With the previous assumptions, the steady-state nucleation rate is:

$$
I_{s s}=N_{0} \sqrt{\frac{\alpha \Delta S_{F}}{R}} \frac{D(T)}{a^{2}} \exp \left(-\frac{\mathcal{C}^{\prime} T^{2}}{\Delta T^{2}}\right)
$$

For homogeneous nucleation, each atom in the liquid acts as a potential nucleation site and $N_{0}=$ $N_{A} / V_{M}$. Such an assumption yields a value of $\tau_{n}^{C N T} \sim 50$ ps at $T=837 \mathrm{~K}$, which is 5 orders of 




FIG. 12. Temperature dependence, in Arrhenius form, of the nucleation time $\tau_{n}$ and structural relaxation time $\tau_{\alpha}$ of $\mathrm{Cu}$ atoms. Solid line is the Vogel-Fulcher-Tammann (VFT) fit of relaxation data. Dash-dotted and dashed lines correspond to Eq. 7 and Eq. 8 respectively, i.e., the predictions of the nucleation time according to CNT. The shaded regions marks the onset temperature $T_{S}$ and the nose temperature $T_{\text {nose }}$ (with their uncertainty).

magnitude smaller than that determined from MD simulations. Note that at this temperature, the estimation of $D(T)$ using or not the fractional SE equation cannot explain such a difference. We emphasize that this value is smaller than that of the relaxation time, giving an unphysical behavior of HNL which would be shifter to higher temperatures with respect to MD simulations.

An alternative approach is to consider that DHs induce a heterogeneous character in the nucleation process. Indeed, we have revealed that the supercooled $\mathrm{Cu}_{5} \mathrm{Zr}$ liquid is not spatially homogeneous, but intrinsically has static structural heterogeneity. More specifically, crystal nucleation is not promoted in the whole volume of the supercooled phase but only in slow regions of DHs and for $\mathrm{Cu}$ atoms with the BOO parameter $q_{6} \sim 0.62$. From an estimation based on the 
different MD runs, we find that at $T=837 \mathrm{~K}, 5 \%$ of particles present in slow regions of DHs can act as a nucleation site. Therefore, the volume fraction of liquid where nucleation process can proceed is drastically reduced and taking into account this effect gives a much higher value for the nucleation time, $\sim 20 \mathrm{~ns}$, as compared to the homogeneous approximation, and not too far from the MD-based value. Note that a similar approach was used in an experimental study of the nucleation rates of several silicate systems [49].

\section{CONCLUSIONS}

In summary, we investigated the role of dynamic heterogeneities in the nucleation kinetics of the supercooled metallic $\mathrm{Cu}_{5} \mathrm{Zr}$ liquid using MD simulations.

- We find that the cross-over temperature $T_{S}$ which marks the onset of DHs and the breakdown of SE relation is located well above the supercooled region where crystal nucleation events are observed. Moreover, at $T_{S}$ we observe the development of local icosahedral symmetry which has a connection to the equilibrium crystal structure.

- Well below $T_{S}$, we find that the underlying nucleation mechanism is clearly not homogeneous since a clear correlation is established between the slow regions of DHs and crystal-like ones. The sequence of crystallization can be then described as follows: (i) inhomogeneous supercooled liquid (ii) slow regions of DHs with a high degree of local icosahedral symmetry acting as nucleation precursors (iii) final crystallization.

- In the framework of CNT, we determine that the occurrence of $\mathrm{HNL}$ at $T$ close to $T_{g}$ is directly related to the decoupling of diffusion from the structural relaxation time. We also propose simple arguments based on DHs to restore the predictive character of CNT for crystal nucleation in deep supercooled liquids.

\section{ACKNOWLEDGEMENTS}

The authors acknowledge the financial support from the Centre of Excellence of Multifunctional Architectured Materials "CEMAM" No ANR-10-LABX-44-01 funded by the "Investments for the Future" Program. This work was granted access to the HPC resources of IDRIS under the allocation 2017-A0020910083 made by GENCI. Some of the computations presented in this

paper were performed using the Froggy platform of the CIMENT infrastructure (https://ciment.ujf- 
grenoble.fr), which is supported by the Rhône-Alpes region and the Equip@Meso project (reference ANR-10-EQPX-29-01) of the programme Investissements d'Avenir supervised by the Agence Nationale pour la Recherche.

[1] J. Fisher, J. Hollomon, and D. Turnbull, J. Appl. Phys 19, 775 (1948).

[2] J. Schroers, Y. Wu, R. Busch, and W. Johnson, Acta Mater. 49, 2773 (2001).

[3] W. L. Johnson, J. H. Na, and M. D. Demetriou, Nat. Commun. 7, 10313 EP (2016).

[4] W. Kauzmann, Chem. Rev. 43, 219 (1948).

[5] H. Tanaka, Phys. Rev. E 68, 011505 (2003).

[6] A. Cavagna, A. Attanasi, and J. Lorenzana, Phys. Rev. Lett. 95, 115702 (2005).

[7] L. Berthier and G. Biroli, Rev. Mod. Phys. 83, 587 (2011).

[8] C. A. Angell, K. L. Ngai, G. B. McKenna, P. F. McMillan, and S.W.Martin, J. Appl. Phys. 88, 3113 (2000).

[9] D. Turnbull, Contemp. Phys. 10, 473 (1969).

[10] E. D. Zanotto and D. R. Cassar, J. Chem. Phys. 149, 024503 (2018).

[11] M. D. Ediger and P. Harrowell, J. Chem. Phys. 137, 080901 (2012).

[12] S. K. Kumar, G. Szamel, and J. F. Douglas, J. Chem. Phys. 124, 214501 (2006).

[13] S. R. Becker, P. H. Poole, and F. W. Starr, Phys. Rev. Lett. 97, 055901 (2006).

[14] K. L. Ngai, J. Phys. Chem. B 103, 10684 (1999).

[15] N. Jakse and A. Pasturel, Phys. Rev. B 94, 224201 (2016).

[16] M. G. Mazza, N. Giovambattista, F. W. Starr, and H. E. Stanley, Phys. Rev. Lett. 96, 057803 (2006).

[17] J. Ding and E. Ma, npj Comput. Mater. 3 (2017).

[18] F. C. Frank, Proc. R. Soc. London 215, 43 (1952).

[19] Y. C. Hu, F. X. Li, M. Z. Li, H. Y. Bai, and W. H. Wang, Nat. Commun. 6, 8310 EP (2015).

[20] G. Tarjus, S. A. Kivelson, Z. Nussinov, and P. Viot, J. Phys.: Condens. Matter 17, R1143 (2005).

[21] H. Tanaka, Eur. Phys. J. E 35, 113 (2012).

[22] Y. Cheng and E. Ma, Progress in Materials Science 56, 379 (2011).

[23] M. Mendelev, M. Kramer, R. Ott, D. Sordelet, D. Yagodin, and P. Popel, Philos. Mag. 89, 967 (2009).

[24] S. Plimpton, J. Comput. Phys. 117, 1 (1995). 
[25] K. Binder and W. Kob, Glassy materials and disordered solids: an introduction to their statistical mechanics (World Scientific, Singapore, 2005).

[26] W. Kob and H. C. Andersen, Phys. Rev. E 51, 4626 (1995).

[27] A. Widmer-Cooper and P. Harrowell, Phys. Rev. Lett. 96, 185701(4) (2006).

[28] P. J. Steinhardt, D. R. Nelson, and M. Ronchetti, Phys. Rev. B 28, 784 (1983).

[29] H. Tanaka, T. Kawasaki, H. Shintani, and K. Watanabe, Nature Materials 9, 324 EP (2010).

[30] M. Leocmach and H. Tanaka, Nature Communications 3, 974 EP (2012).

[31] F. H. Stillinger and T. A. Weber, Phys. Rev. A 25, 978 (1982).

[32] J. Orava and A. L. Greer, J. Chem. Phys. 140, 214504 (2014).

[33] F. Puosi, N. Jakse, and A. Pasturel, J. Phys.: Condens. Matter 30, 145701 (2018).

[34] P. R. ten Wolde, M. J. Ruiz-Montero, and D. Frenkel, The Journal of Chemical Physics 104, 9932 (1996).

[35] $\mathrm{Cu}_{5} \mathrm{Zr}$ crystal has a cubic $\mathrm{Be}_{5} \mathrm{Au}$-type crystal structure (space group $F-43 m$ ). In the 24- atoms unit cell, $16 \mathrm{Cu}$ atoms (Cu2) occupy equivalent sites with an icosahedral environment while the 4 other $\mathrm{Cu}(\mathrm{Cu} 1)$ and the $4 \mathrm{Zr}$ atoms occupy sites with a similar complex atomic environment consisting of a 16-vertex Frank-Kasper polyhedron.

[36] E. V. Levchenko, A. V. Evteev, I. V. Belova, and G. E. Murch, Acta Mater. 59, 6412 (2011).

[37] P. K. Gupta, D. R. Cassar, and E. D. Zanotto, J. Chem. Phys. 145, 211920 (2016).

[38] F. Spaepen, Acta Metall. 23, 729 (1975).

[39] F. Spaepen and R. B. Meyer, Scr. Metall. 10, 257 (1976).

[40] D. Holland-Moritz, Int. J. Non-Equilib. Process. 11, 169 (1998).

[41] K. F. Kelton, G. W. Lee, A. K. Gangopadhyay, R. W. Hyers, T. J. Rathz, J. R. Rogers, M. B. Robinson, and D. S. Robinson, Phys. Rev. Lett. 90, 195504 (2003).

[42] G. W. Lee, A. K. Gangopadhyay, T. K. Croat, T. J. Rathz, R. W. Hyers, J. R. Rogers, and K. F. Kelton, Phys. Rev. B 72, 174107 (2005).

[43] D. Turnbull, J. Appl. Phys 21, 1022 (1950).

[44] K. Yamaguchi, Y.-C. Song, T. Yoshida, and K. Itagaki, J. Alloys Compd. 452, 73 (2008).

[45] K. Kelton and A. Greer, Nucleation in Condensed Matter: Applications in Materials and Biology, Pergamon Materials Series, Vol. 15 (Pergamon, 2010).

[46] I. S. Gutzow and J. W. Schmelzer, The Vitreous State: Thermodynamics, strucutre, rheology, and crystallization (Springer-Verlag Berlin Heidelberg, 2014). 
[47] V. M. Fokin, E. D. Zanotto, N. S. Yuritsyn, and J. W. Schmelzer, J. Non-Cryst. Solids 352, 2681 (2006).

[48] P. G. Debenedetti, Metastable liquids: concepts and principles (Princeton University Press, 1997).

[49] A. S. Abyzov, V. M. Fokin, N. S. Yuritsyn, A. M. Rodrigues, and J. W. Schmelzer, J. Non-Cryst. Solids 462, 32 (2017). 

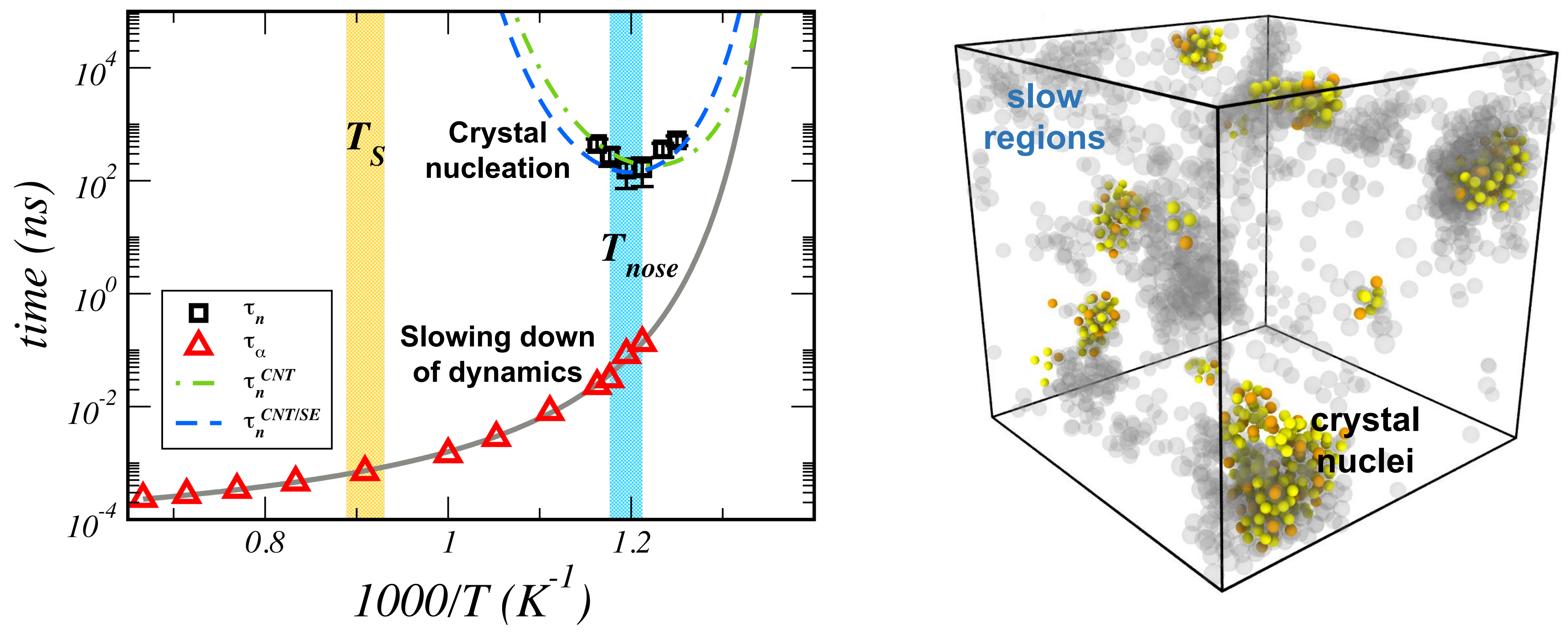